\title{
DEVELOPMENT AND APPLICATIONS OF THE GAS ELECTRON MULTIPLIER
}

\author{
S. Bachmann ${ }^{1}$, A. Bressan ${ }^{1}$, S. Kappler ${ }^{2}$, B. Ketzer ${ }^{1}$, M. Deutel ${ }^{1}$, \\ L. Ropelewski ${ }^{1}$, F. Sauli ${ }^{1}$ and E. Schulte ${ }^{3}$ \\ 1 CERN, Geneva, Switzerland \\ 2 Karlsruhe University, Germany \\ 3 Helsinki University, Finland
}

\begin{abstract}
The Gas Electron Multiplier (GEM) has been recently developed to cope with the severe requirements of high luminosity particle physics experimentation. With excellent position accuracy and very high rate capability, GEM devices are robust and easy to manufacture. The possibility of cascading two or more multipliers permits to achieve larger gains and more stable operation. We discuss major performances of the new detectors, particularly in view of possible use for high rate portal imaging and medical diagnostics.
\end{abstract}

Keywords: Gas Electron Multiplier, GEM, gas detector, medical imaging 
Imaging 2000 conference

Stockholm, Sweden, June 28-July 1, 2000

Corresponding author: fabio.sauli@cern.ch 


\section{Introduction}

Following the innovative invention of the micro-strip gas chamber (MSGC [1]), a variety of performing gaseous devices, collectively named micropattern detectors (MPD), has been developed in the recent years [2]. Providing good detection efficiency and localization accuracy at high rates, they are successfully used in high energy physics experiments and other applied fields. Recent experience has shown however that MPDs have a tendency to discharge when exposed to high rates or to highly ionizing particles [3-5]. The appearance of discharges is attributed to the avalanche size exceeding a critical value, in the range $10^{7}-10^{8}$, the so-called Raether limit. At this point, a transition from proportional avalanche to streamer may occur, often followed by a rupture of the gas dielectric rigidity. With the exception of the MSGC, the new MPD devices are generally sturdy enough to withstand discharges without damages. This may not be the case however for the sensitive readout electronics; moreover, the recovery time after a breakdown can seriously affects the detector efficiency.

\section{The gas electron multiplier}

The recently introduced gas electron multiplier (GEM [6]) offers a way for improvement on this crucial point. The basic component of the detector is a metal-coated thin insulating foil, chemically pierced with a high density of holes, typically 50-100 $\mu \mathrm{m}$ in diameter at $100-200 \mu \mathrm{m}$ pitch ${ }^{1}$. With proper choice of the applied potentials, electrons released in the gas above the foil are drifted into the holes, and multiplied in avalanche in the high field within the channels (Fig. 1). Most of the electrons in the avalanche move into the lower gap, and can be collected on a passive electrode. Effective gains well above several thousand have been achieved [7, 8]. Spatial localization can then be obtained collecting the charge on a patterned one- or two-dimensional readout board; typical position accuracy around $40 \mu \mathrm{m}$ rms have been obtained for tracks and soft X-rays [9].

A unique feature of GEM detectors is that proportional multiplication and charge detection are performed on separate electrodes. With proper choice of the operating conditions, one can effectively avoid the propagation of accidental discharges to the sensitive electronics [10,11]. The structure of the readout plane itself can be easily adapted to the experimental needs, with pads or strips of arbitrary shapes. The signals are purely due to electrons, without ion tails, and are therefore very fast. Using thin multi-layer boards, all coordinates can be kept at ground potential, resulting in a considerable simplification in the read-out electronics.

\section{Multi-step devices}

The gas electron multiplier makes no exception to the above-mentioned

\footnotetext{
${ }^{1}$ GEM and read-out plane manufacturing technologies developed by A. Gandi and R. De Oliveira (CERN-EST-SM)
} 
appearance of discharges at high rates or in presence of heavily ionizing tacks. A solution to this fundamental problem has been found however, by cascading several multiplying elements, an approach developed long ago in the so-called multi-step chamber [12]. This can be easily achieved with a GEM, used as pre-amplifier in combination with another MPD. The exact reasons for the improvement are not completely clear. It is suspected that, since in cascaded system the same overall gain is obtained with each element operated at reduced voltage, the amount of charge required for the transition is larger. In other words, the Raether limit might be voltage dependent. Alternatively, one can invoke the larger avalanche spread in multiple structures to reduce the charge density [4]. A two-stage detector made with a GEM in front of a MSGC permits to reach an order of magnitude higher gains before discharges $[7,13]$. This is the solution adopted for the tracker of HERAB experiment, originally based on the MSGC technology and has led to the first large production of GEM electrodes. More than two hundred foils, 27x25 $\mathrm{cm}^{2}$ each, have been manufactured and are being integrated in the experimental set-up at DESY [14].

Similar performances are obtained with multiple GEM devices, intrinsically cheaper and more robust than MSGCs [15]. Double GEM detectors (Fig. 2) have been extensively tested with soft X-rays and in particle beams [16]. With a double GEM, gains in excess of $10^{4}$ can be reached at very high rates, see Fig. 3 [3]. On exposure to highly ionizing alpha particles, the maximum safe gain is about an order of magnitude larger than for a single structure. Three and four-stage GEMs are under development for the use at LHC [17] and to achieve single photoelectron detection [18]. A system of large size (31x31 $\mathrm{cm}^{2}$ active) double-GEM detectors is in construction at CERN for the needs of the COMPASS experiment; prototypes have been successfully tested in realistic experimental conditions [19]. Fig. 4 shows one detector installed in a high intensity beam at the Paul Scherrer Institut in Villigen.

\section{Two-dimensional readout and imaging with GEM detectors}

GEM detectors can be easily equipped for two-dimensional read-out, sharing the collected electrons in the last gap between two sets of perpendicular, partly overlapping strips; this is the solution adopted for the COMPASS chambers. Exploiting also the induced signal on the lower electrode of the GEM closer to the read-out board, one can trigger the charge recording electronics on neutral events and realize two-dimensional maps of X-ray activity [9]. Fig. 5 gives an example of X-ray absorption radiography of a small mammal, recorded with a double GEM detector; the image size is about $6 \times 3 \mathrm{~cm}^{2}$. The intrinsic space resolution is better than $100 \mu \mathrm{m}, \mathrm{a}$ promising feature for possible medical applications.

The high rate capability (well above $10^{5} \mathrm{~Hz} \mathrm{~mm}^{-2}$ ), the good position accuracy, robustness and radiation hardness makes GEM detectors good candidates for digital medical diagnostics and portal imaging. A threatening problem concerns however the implementation of a suitable read-out electronics. Most existing high-density read-out systems, developed for particle physics, have a high input rate capability, but a limited serial transfer rate of selected events. Moreover, with an intrinsic time resolution per event 
of around hundred ns, the commonly used 2-dimensional projective readout scheme would generate too many ambiguities at rates exceeding few hundred $\mathrm{kHz}$ over the detector area. A pixel read-out is of course the most powerful scheme for these applications, and its implementation is made possible by the freedom of choice of the charge collecting electrodes in GEM detectors. Highdensity pixel readout electronics is under development for detectors in the core of high luminosity experiments. To preserve localization accuracy, however, the pixel size should not exceed half a $\mathrm{mm}$ or so, with a consequent inflation in the number of required channels.

An elegant alternative, offering performances between projective strips and pixels, is being developed under the name of hexaboard [9, 10]. As shown in Fig. 6, it consists in a matrix of charge-collecting hexagonal pixels, interconnected on the backside in rows along three directions at $60^{\circ}$ to each other $^{2}$. For each event, three independent charge profiles are recorded, providing an ambiguity-free reconstruction for most multiple events. A 10x10 $\mathrm{cm}^{2}$ double GEM detector with the new read-out pattern has been tested, demonstrating localization properties very similar to the more conventional 2-D projective strip readout. The ambiguity resolution power of the hexaboard readout has been verified adding up by software events collected at low rate (due to the present electronics limitations). The results are shown in Fig. 7. On the left side, the original X-ray absorption image obtained, for single events, with a finely segmented mask, $8 \mathrm{~mm}$ on the side; taking into account the two-track resolution in each projection, around one $\mathrm{mm}$, the corresponding occupancy is $12 \%$. On the right, the same image reconstructed after random addition of pairs of events to simulate double tracks; the efficiency is about to $70 \%$, despite the rather primitive algorithm used for the analysis.

\section{Single photon detection and localization}

The confinement of avalanches in the holes, with the consequent suppression of photon feedback, probably explains the exceptionally large gains obtained operating the device in pure noble gases and their mixtures, see Fig. 8 [20]. The absence of organic quenchers, often required in other structures to achieve high gains, should easy the use of alkali photocathode materials. Applications include the development of large area, positionsensitive gas photo-multipliers. Various gain-limiting feedback processes are being analyzed in order to optimize the device [21]; recent studies have focussed on the optimization of multiple GEM structures for the efficient detection of single photoelectrons [22, 23]. A very attractive possibility is to use the upper GEM electrode, facing a transparent window, as photocathode, followed by a transfer of the photoelectrons through the holes to another gas amplifying device. The strong suppression of photon and ion feedback in this reverse photocathode configuration should results in easy single photon detection and extended lifetime of the detector.

\footnotetext{
${ }^{2}$ Developed by R. De Oliveira (CERN-EST-SM)
} 
The part of this work describing the imaging performances of GEM has greatly profited from continuing support and interest of the group led by Prof. T. Müller (Inst. Experimentelle Kernphysik, Universität Karlsruhe). 


\section{REFERENCES}

[1] A. Oed, Nucl. Instrum. and Meth. A263 (1988) 351.

[2] F. Sauli and A. Sharma, Ann. Rev. Nucl. Part. Sci. 49 (1999) 341.

[3] A. Bressan, M. Hoch, P. Pagano, L. Ropelewski, F. Sauli, S. Biagi, A. Buzulutskov, M. Gruwé, A. Sharma, D. Moermann, G. De Lentdecker, Nucl. Instrum. and Meth. A424 (1998) 321.

[4] V. Peskov, B.D. Ramsey, P. Fonte, IEEE Trans. Nucl. Sci. NS-45 (1998) 244.

[5] P. Fonte, V. Peskov, B.D. Ramsey, Nucl. Instrum. and Meth. A 419 (1998) 405.

[6] F. Sauli, Nucl. Instrum. and Meth. A386 (1997) 531.

[7] R. Bouclier, W. Dominik, M. Hoch, J.C. Labbé, G. Million, L. Ropelewski, F. Sauli, A. Sharma, G. Manzin, Nucl. Instrum. and Meth. A396 (1997) 50.

[8] J. Benlloch, A. Bressan, M. Capeáns, M. Gruwé, M. Hoch, J.C. Labbé, A. Placci, L. Ropelewski, F. Sauli, Nucl. Instrum. and Meth. A 419 (1998) 410.

[9] A. Bressan, L. Ropelewski, F. Sauli, D. Mörmann, T. Müller, H.J. Simonis, Nucl. Instrum. and Meth. A425 (1999) 254.

[10]F. Sauli, Gas detectors: achievements and trends, 8th Pisa Meeting on Advanced Detectors (Isola d'Elba, Italy, 21-27 May 2000). CERN-EP/2000080. Subm. Nucl. Instrum. Methods (2000).

[11]S. Bachmann, A. Bressan, M. Deutel, S. Kappler, B. Ketzer, A. Polouektov, L. Ropelewski, F. Sauli, E. Schulte, L. Shekhtman, A. Sokolov, Discharge mechanisms and prevention in the gas electron multiplier, Subm. Nucl. Instrum. Methods (2000).

[12] G. Charpak and F. Sauli, Phys. Letters 78 B (1978) 523.

[13]Y. Benhammou, R. Blaes, J.M. Brom, F. Drouhin, J.C. Fontaine, D. Huss, F. Jeanneau, V. Mack, I. Ripp-Baudot, B. Schwaller, A. Zghiche, Nucl. Instrum. Methods A441 (2000) 452.

[14] T. Zeuner, Nucl. Instrum. Methods A 446 (2000) 324.

[15]C. Büttner, M. Capeáns, W. Dominik, M. Hoch, J.C. Labbé, G. Manzin, G. Million, L. Ropelewski, F. Sauli, A. Sharma, Nucl. Instrum. and Meth. A 409 (1998) 79.

[16]A. Bressan, J.C. Labbé, P. Pagano, L. Ropelewski, F. Sauli, Nucl. Instrum. and Meth. A425 (1999) 262.

[17] M. Ziegler, P. Cwetanski, U. Straumann, A triple GEM detector for LHCb, LHCb TRAC 99-024 (1999).

[18]G. Garty, R. Chechik, A. Breskin, E. Shefer, Nucl. Instrum. and Meth. A433 (1999) 476.

[19]S. Bachmann, A. Bressan, A. Placci, L. Ropelewski, F. Sauli, IEEE Trans. Nucl. Sci. NS-47 (2000) .

[20] A. Buzulutskov, A. Breskin, R. Chechik, G. Garty, F. Sauli, L. Shekhtman, Nucl. Instrum. Methods A 443 (2000) 164.

[21]A. Breskin, T. Boutboul, A. Buzulutskov, R. Chechik, G. Garty, E. Shefer, B.K. Singh, Nucl. Instrum. Methods A 442 (2000) 58.

[22]A. Sharma, Detection of single electrons emitted by internal photocathodes with the gas electron multiplier (GEM), CERN-OPEN99/372. Subm. Nucl. Instrum. Methods (1999). 
[23]C. Richter, A. Breskin, R. Chechik, G. Garty, A. Sharma, Single electron transfer efficiency of GEM, 8th Pisa Meeting on Advanced Detectors (Isola d'Elba, Italy, 21-27 May 2000). Subm. Nucl. Instrum. Methods (2000). 


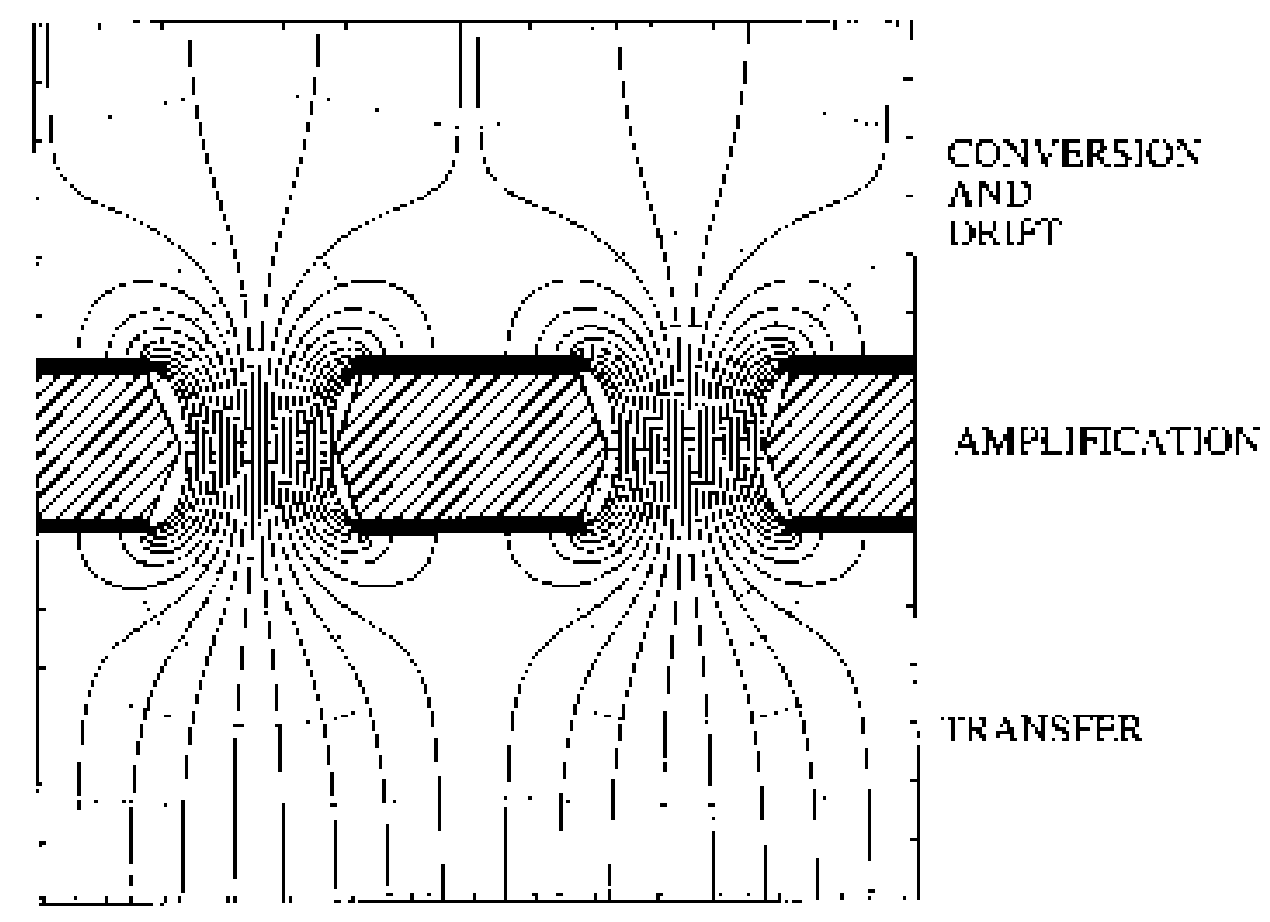

Fig. 1: Schematics and fields of the gas electron multiplier.

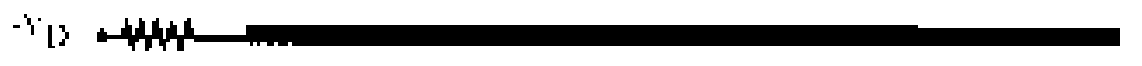

$r_{-I J} \quad|>|\langle I):$

$-v_{i}:$

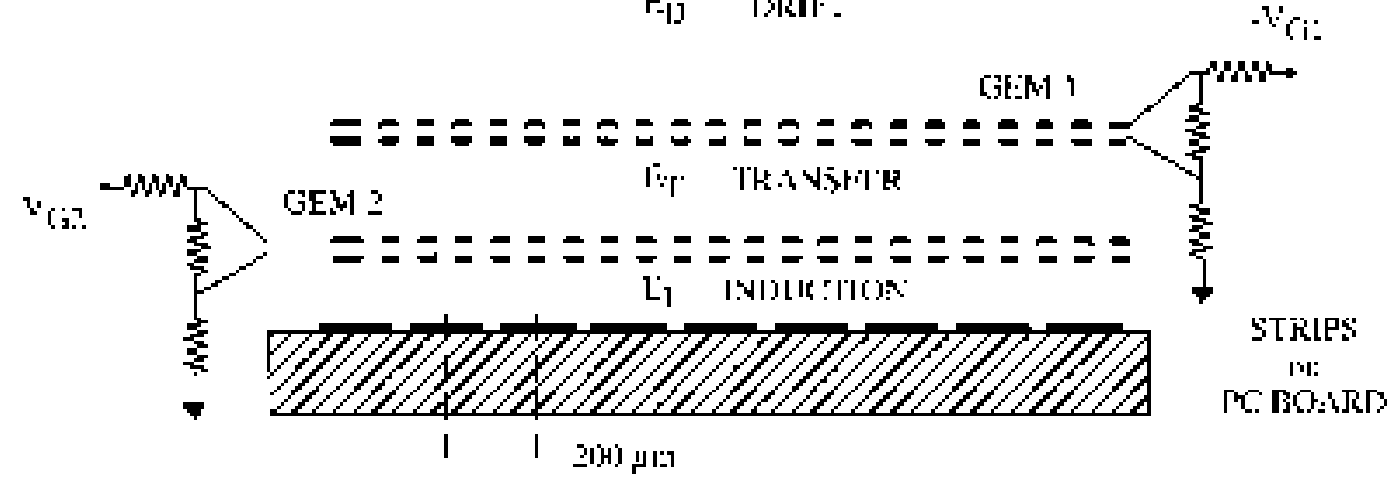

Fig. 2: Schematics of the double-GEM detector. 


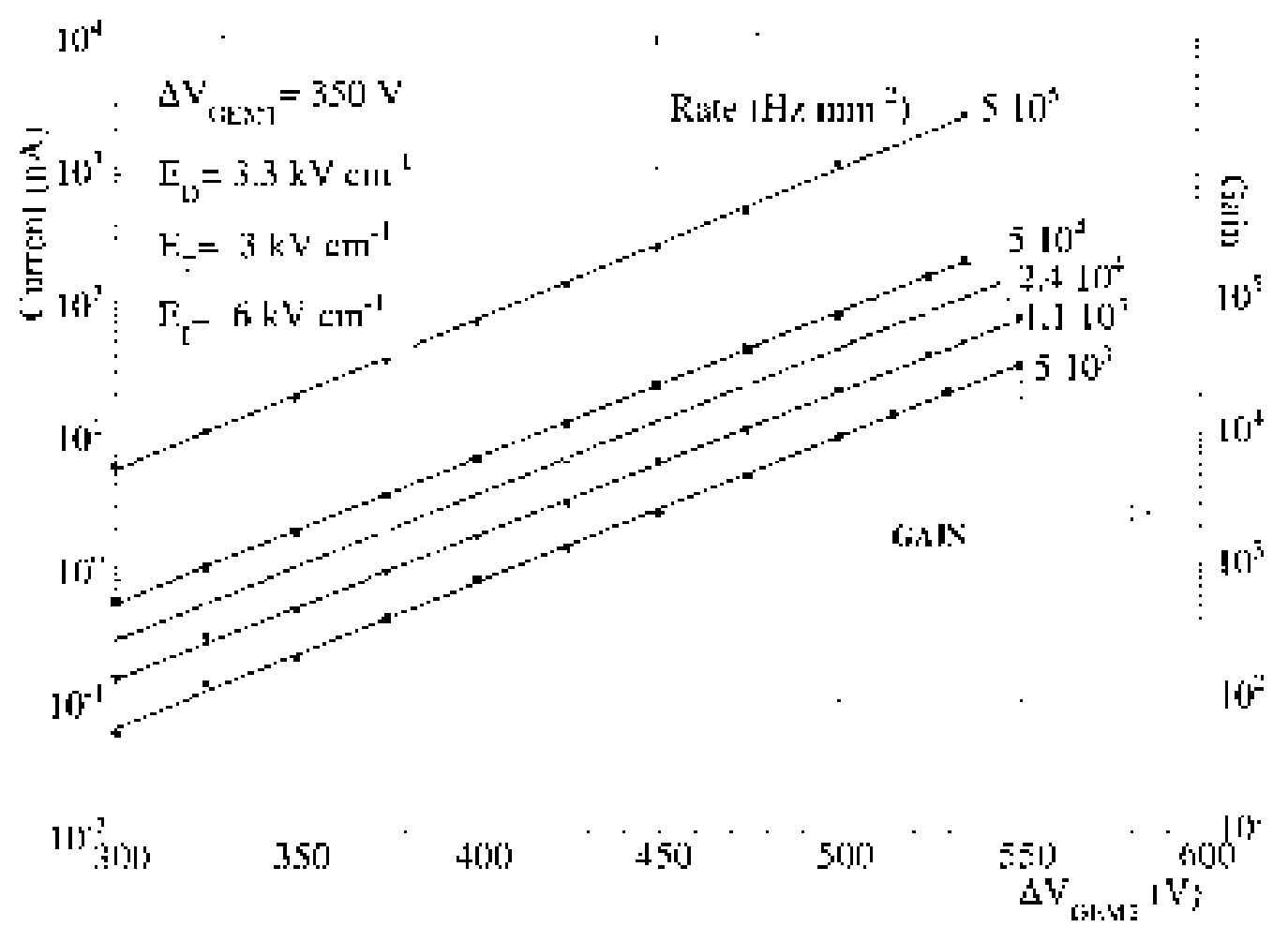

Fig. 3: Current in the double-GEM detector as a function of voltage in one GEM, for increasing X-ray flux. The gain can be read on the lower curve.

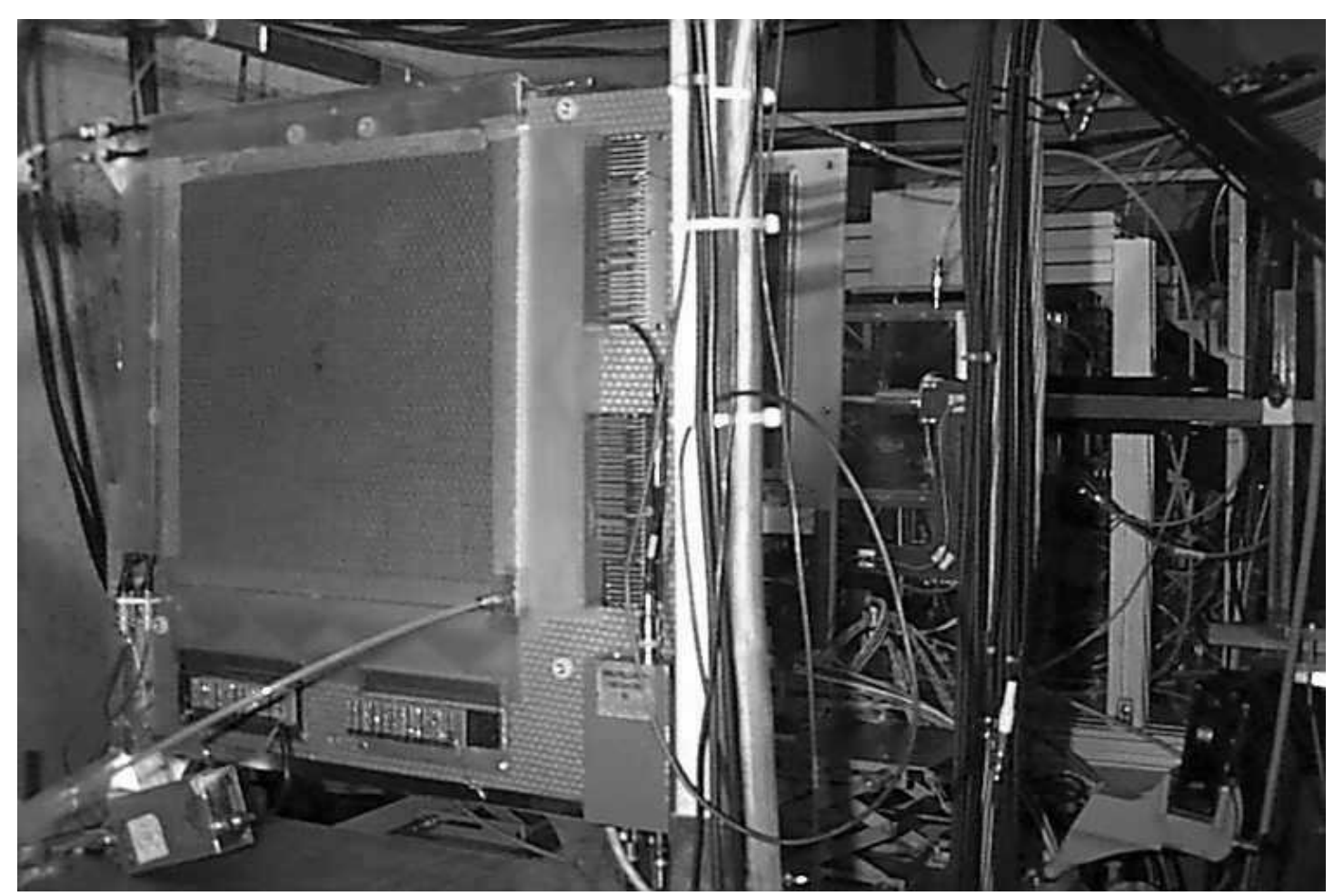


Fig. 4: A full-size prototype for COMPASS installed in the PSI beam.

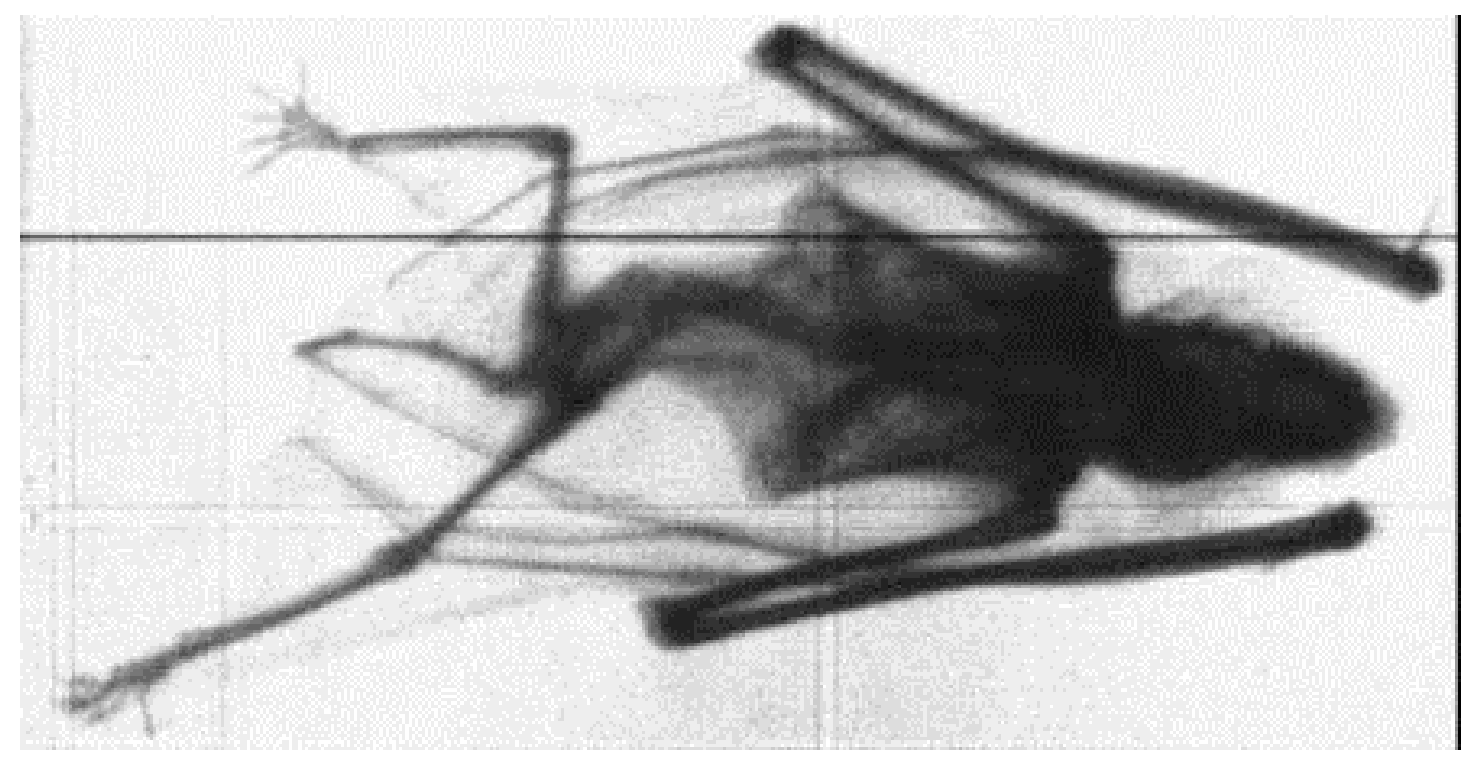

Fig. 5: $8 \mathrm{keV}$ absorption radiography of a small mammal. The horizontal image size is about $6 \mathrm{~cm}$.

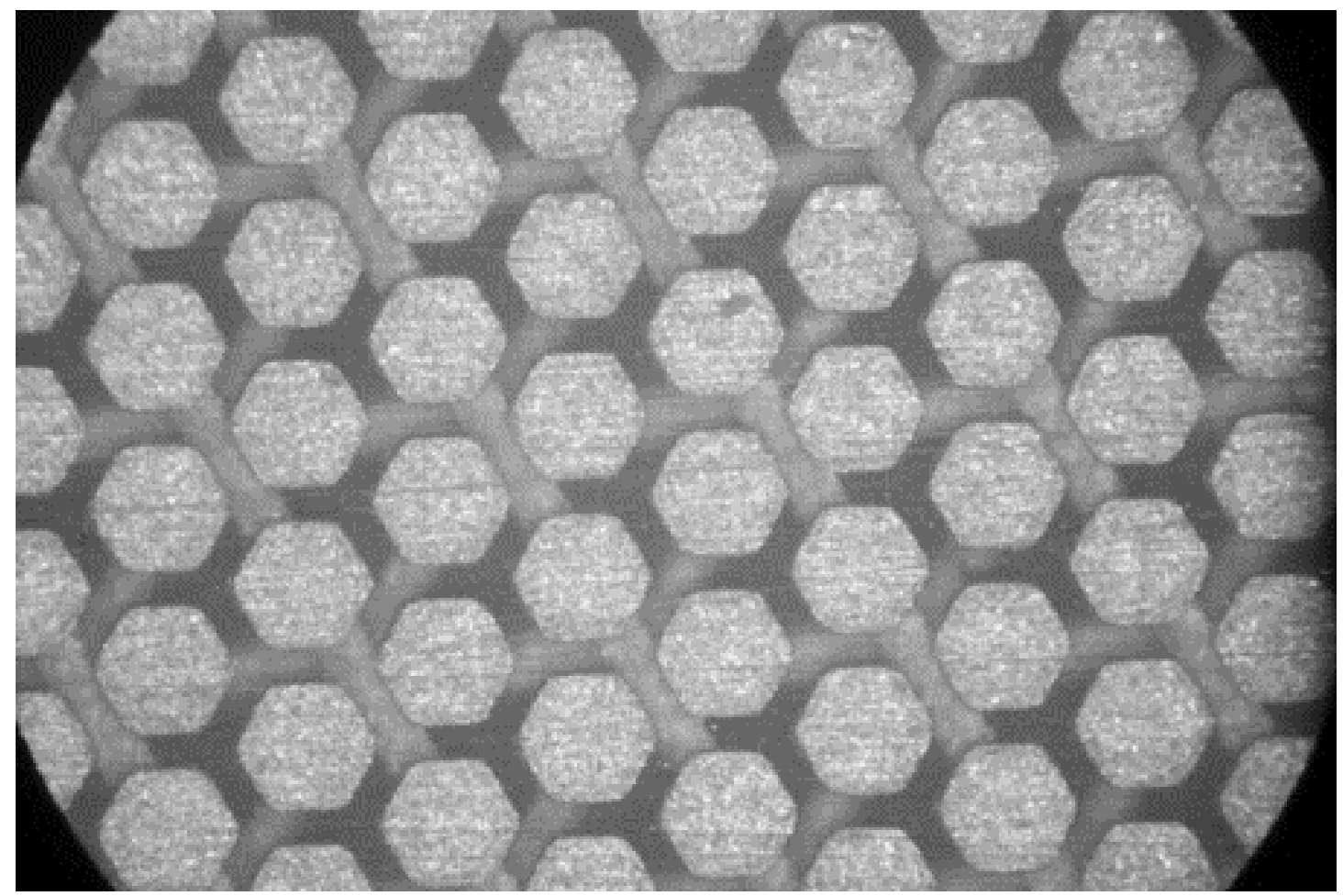


Fig. 6: Close view of the hexaboard readout plane: pads are $500 \mu \mathrm{m}$ in diameter, interconnected along three directions at $60^{\circ}$.
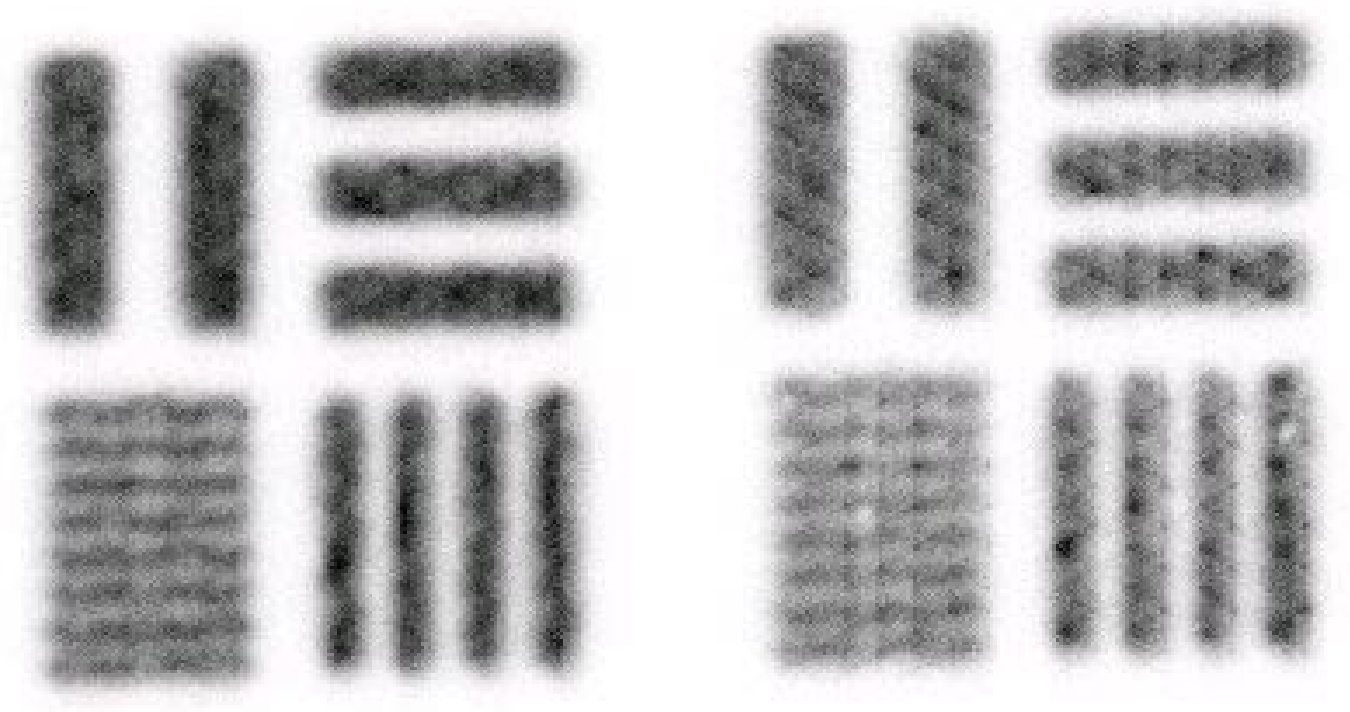

Fig. 7: Two-dimensional X-ray absorption pattern with a mask, $1 \mathrm{~mm}$ on the side, recorded with the hexaboard chamber. Left: the original pattern. Right: the one obtained adding up pairs of events to simulate high rates.

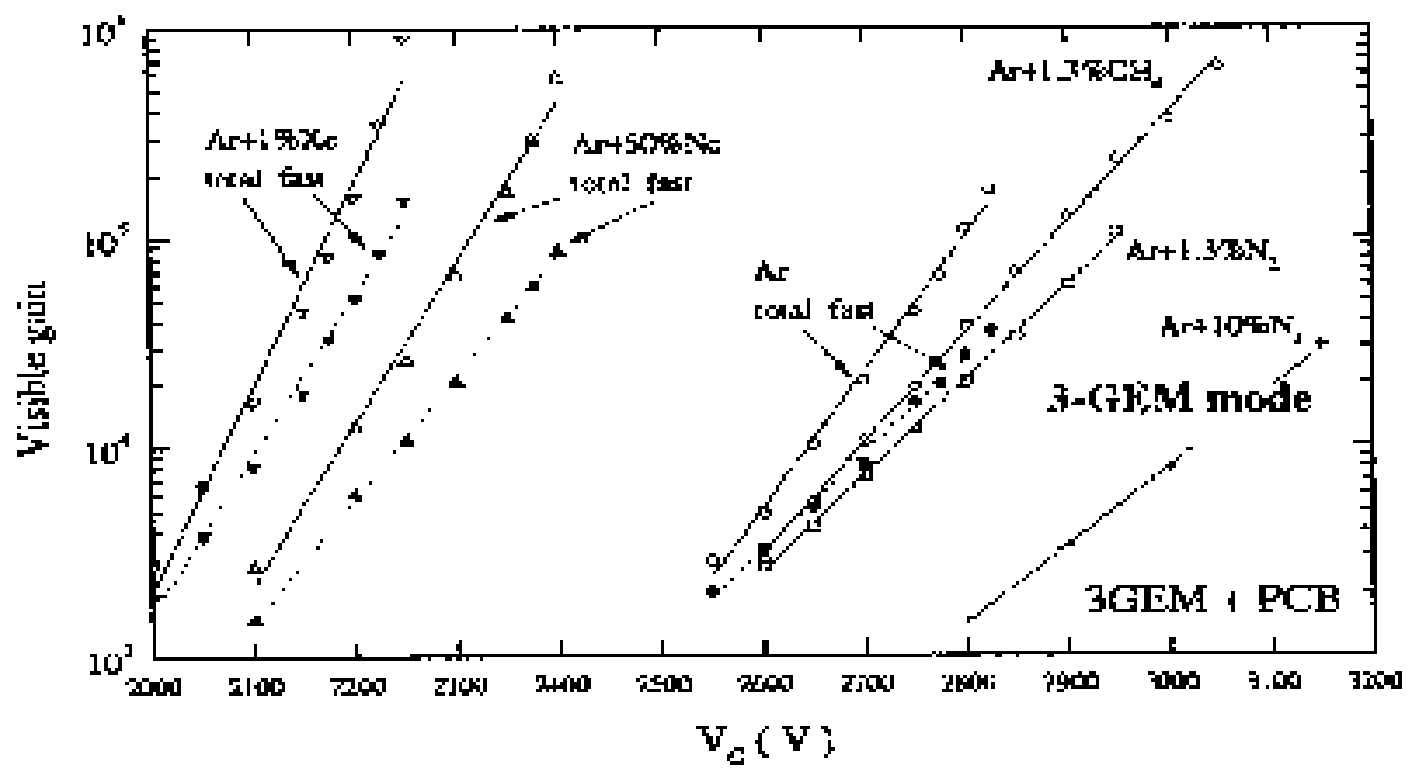


Fig. 8: Large gains obtained with triple-GEM structure in various noble gas mixtures. 